九州大学学術情報リポジトリ

Kyushu University Institutional Repository

\title{
ON NONDETERMINISTIC DYNAMIC PROGRAMMING
}

Hisano, Hiroshi

Department of Information, Tohwa University

https://doi.org/10.5109/18991

出版情報: Bulletin of informatics and cybernetics. 40, pp.1-15，2008-12. Research Association of Statistical Sciences

バージョン :

権利関係 : 
ON NONDETERMINISTIC DYNAMIC PROGRAMMING

by

Hiroshi Hisano

Reprinted from the Bulletin of Informatics and Cybernetics

Research Association of Statistical Sciences, Vol.40

FUKUOKA, JAPAN

2008 


\title{
ON NONDETERMINISTIC DYNAMIC PROGRAMMING
}

\author{
By
}

\section{Hiroshi Hisano*}

\begin{abstract}
R. Bellman left a lot of research problems in his work "Dynamic Programming" (1957). Having received ideas from Bellman, S. Iwamoto has extracted, out of his problems, a problem on nondeterministic dynamic programming (NDP). Instead of stochastic dynamic programming which has been well studied, Iwamoto has opened a gate to NDP. This report presents specific optimal solutions for NDPs on continuous state and decision spaces.
\end{abstract}

Key Words and Phrases: nondeterministic, dynamic programming, controlled integral equation, unbounded transition weight, continuous state and decision

\section{Introduction}

R. Bellman (1957) has proposed 373 Exercises and Research Problems in all. Some of Research Problems are resolved. Some are still unsolved. Among unsolved ones, there are a few interesting dynamic programming (DP) problems in Bellman (1957; pp.124125, pp.132-133). They are neither deterministic nor stochastic. Fujita et al. (2004) and Iwamoto (2005) has called them nondeterministic. The nondeterministic implies stochastic, and stochastic does deterministic. Both stochastic DP and deterministic DP are widely applied in science, engineering, economics and others. However, nondeterministic dynamic programming (NDP) is a new research problem (Iwamoto (2005)). This paper treats a class of NDP problems on continuous space. Fujita et al. (2004) have presented a finite discrete NDP model, Hisano (2003) has solved an optimal nondeterministic stopping problems on finite state and decision spaces, and Hisano (2007) has solved a stopping problem on tree, which is also viewed as a nondeterminstic DP.

In this paper we consider both finite- and infinite-stage DP problems with nondeteministic transition law on one-dimensional state and decision spaces. The term nondeterministic is used here in the following meaning. If a decision maker adopts a decision $u(0<u<x)$ on state $x(>0)$, next state $y(0<y \leq u)$ appears with a transition weight $p(y)=\frac{1}{y}$. The weight function $p$ does not satisfy the total unit property $\int_{0}^{u} p(y) d y=1$. Since this function is divergent to $\infty$ as $y$ approaches 0 , it does $\int_{0}^{u} p(y) d y=\infty$. Thus the weight function $p(y)=\frac{1}{y}$ is no more stochastic.

We discuss a three-stage NDP in Section 2, an $n$-stage NDP in Section 3 and an infinite-stage NDP in Section 4. In a stationary model, an optimal policy is shown in the class of stationary policies. In Section 5 , we examine related problems with a discount factor. In Section 6 we show that there is no appropriate weight other than $p(y)=\frac{1}{y}$.

\footnotetext{
* Department of Information, TOHWA University Fukuoka 541-1152, Japan. tel +81-92-541-1152 his@tohwa-u.ac.jp
} 


\section{Three-stage NDP}

In this section we consider the following minimization problem:

Problem 1

Minimize $\quad c(u(x))^{m}+d(x-u(x))^{m}+\int_{C} \frac{m \alpha\left[c(v(y))^{m}+d(y-v(y))^{m}\right]}{y} d y$

$$
+\iint_{D} \frac{(m \alpha)^{2}\left[c(w(z))^{m}+d(z-w(z))^{m}\right]}{y z} d y d z+\iiint_{E} \frac{(m \alpha)^{3} k a^{m}}{y z a} d y d z d a
$$

subject to $\quad 0<u(x) \leq x, 0<v(y) \leq y, 0<w(z) \leq z, u, v, w$ : continuous

where $m$ is a positive integer and the integral domains are

$$
\begin{aligned}
& C=\{y \mid 0<y<u(x)\} \subset(0, \infty) \\
& D=\{(y, z) \mid 0<y<u(x), 0<z<v(y)\} \subset(0, \infty)^{2} \\
& E=\{(y, z, \alpha) \mid 0<y<u(x), 0<z<v(y), 0<\alpha<w(z)\} \subset(0, \infty)^{3} .
\end{aligned}
$$

We have recourse to the following lemmas to solve problems in this paper.

LEMMA 2.1. Let $m$ be a positive integer. Then the following two problems $\left(\mathrm{P}_{1}\right)$ and $\left(\mathrm{P}_{2}\right)$ are essentially identical.

$$
\begin{array}{cl}
\text { Minimize } & c u(x)^{m}+d(x-u(x))^{m}+m \alpha \int_{C} \frac{k y^{m}}{y} d y \\
\text { subject to } & 0<u(x) \leq x, u: \text { continuous, where } C=\{y \mid 0<y<u(x)\}
\end{array}
$$

$$
\begin{aligned}
\text { Minimize } & c u^{m} x^{m}+d(x-u x)^{m}+m \alpha \int_{C} \frac{k y^{m}}{y} d y \\
\text { subject to } & 0<u \leq 1, \text { where } C=\{y \mid 0<y<u x\} .
\end{aligned}
$$

Proof. The objective of problem $\left(\mathrm{P}_{1}\right)$ lies with finding the function $u(x)$ and to this end it suffices to show that $u(x)$ can be indicated in the form of an linear expression $u x$. We have

$$
c u(x)^{m}+d(x-u(x))^{m}+m \alpha \int_{C} \frac{k y^{m}}{y} d y=(c+\alpha k) u(x)^{m}+d(x-u(x))^{m} .
$$

Since $x$ is fixed, this problem means to minimize the function of $m$-th degree

$$
(c+\alpha k) t^{m}+d(x-t)^{m}
$$

with respect to $t$. Differentiating $(c+\alpha k) t^{m}+d(x-t)^{m}$ with respect to $t$ and setting it zero, we get

$$
t=\frac{\sqrt[m-1]{d}}{\sqrt[m-1]{c+\alpha k}+\sqrt[m-1]{d}} x
$$


Let us set

$$
u=\frac{\sqrt[m-1]{d}}{\sqrt[m-1]{c+\alpha k}+\sqrt[m-1]{d}}
$$

Then $u(x)$ takes a linear form $u x$.

LEMMA 2.2. Let $m$ be a positive integer. Then the following two problems $\left(\mathrm{P}_{3}\right)$ and $\left(\mathrm{P}_{4}\right)$ are essentially identical.

$\left(\mathrm{P}_{3}\right)$

$$
\text { Minimize } \quad c u(x)^{m}+d(x-u(x))^{m}+m \alpha \int_{C} \frac{c(v(y))^{m}+d(y-v(y))^{m}}{y} d y
$$

$$
\text { subject to } \quad 0<u(x) \leq x, u \text { : continuous, where } C=\{y \mid 0<y<u(x)\}
$$

$$
\text { Minimize } \quad c u^{m} x^{m}+d(x-u x)^{m}+m \alpha \int_{C} \frac{c v^{m} y^{m}+d(y-v y)^{m}}{y} d y
$$

subject to $0<u \leq 1$, where $C=\{y \mid 0<y<u x\}$.

PRoOF. We can prove Lemma 2.2 by the same way of thinking as Lemma 2.1.

We solve all problems with the following plan using Lemma 2.1 and Lemma 2.2 everywhere:

1. Suppose that we have already selected $u(x)$ and $v(y)$, we decide $w(z)$.

2. After that, we take the same procedure by backward induction of dynamic programming.

\subsection{The solution of Problem 1}

First we have

$$
\begin{aligned}
& (m \alpha)^{2} \iint_{D} \frac{c w(z)^{m}+d(z-w(z))^{m}}{y z} d y d z+(m \alpha)^{3} \iiint_{E} \frac{k a^{m}}{y z a} d y d z d a \\
= & (m \alpha)^{2} \int_{0}^{u(x)}\left[\frac{1}{y} \int_{0}^{v(y)}\left\{\frac{c w(z)^{m}+d(z-w(z))^{m}}{z}+\frac{k \alpha w(z)^{m}}{z}\right\} d z\right] d y .
\end{aligned}
$$

Consider

$$
\int_{0}^{v(y)}\left\{\frac{c w(z)^{m}+d(z-w(z))^{m}}{z}+\frac{k \alpha w(z)^{m}}{z}\right\} d z
$$

Here, the integral function is calculated as follows:

$$
\frac{c w(z)^{m}+d(z-w(z))^{m}}{z}+\frac{k \alpha w(z)^{m}}{z}=\frac{(c+k \alpha) w(z)^{m}+d(z-w(z))^{m}}{z} .
$$

By Lemma 2.1 we can consider the last term as

$$
\left\{(c+k \alpha) w^{m}+d(1-w)^{m}\right\} z^{m-1},
$$


and the minimum point is

$$
\hat{w}=\frac{\sqrt[m-1]{d}}{\sqrt[m-1]{c+\alpha k}+\sqrt[m-1]{d}}
$$

Second we have

$$
\begin{aligned}
& c(u(x))^{m}+d(x-u(x))^{m}+\int_{C} \frac{m \alpha\left[c(v(y))^{m}+d(y-v(y))^{m}\right]}{y} d y \\
+ & \iint_{D} \frac{(m \alpha)^{2}\left[c(w(z))^{m}+d(z-w(z))^{m}\right]}{y z} d y d z+\iiint_{E} \frac{(m \alpha)^{3} k a^{m}}{y z a} d y d z d a \\
= & c(u(x))^{m}+d(x-u(x))^{m}+\int_{C} \frac{m \alpha\left[c(v(y))^{m}+d(y-v(y))^{m}\right]}{y} d y \\
& +(m \alpha)^{2} \int_{0}^{u(x)}\left[\frac{1}{y} \int_{0}^{v(y)}\left\{\frac{c w(z)^{m}+d(z-w(z))^{m}}{z}+\frac{k \alpha w(z)^{m}}{z}\right\} d z\right] d y \\
\geq & c(u(x))^{m}+d(x-u(x))^{m}+\int_{C} \frac{m \alpha\left[c(v(y))^{m}+d(y-v(y))^{m}\right]}{y} d y \\
& +(m \alpha)^{2} \int_{0}^{u(x)}\left[\frac{1}{y} \int_{0}^{v(y)}\left\{\frac{\left.(c+k \alpha) \hat{w}^{m} z^{m}+d(1-\hat{w})^{m} z^{m}\right)}{z}\right\} d z\right] d y \\
= & \left.c(u(x))^{m}+d(x-u(x))^{m}\right] \\
& +(m \alpha) \int_{0}^{u(x)}\left\{c v^{m}+d(1-v)^{m}+\alpha\left\{(c+k \alpha) \hat{w}^{m}+d(1-\hat{w})^{m}\right\} v^{m}\right\} y^{m-1} d y
\end{aligned}
$$

Set

$$
k_{1}=\left\{(c+k \alpha) \hat{w}^{m}+d(1-\hat{w})^{m}\right\},
$$

and we have

$$
(1)=c(u(x))^{m}+d(x-u(x))^{m}+(m \alpha) \int_{0}^{u(x)}\left\{c v^{m}+d(1-v)^{m}+k_{1} \alpha v^{m}\right\} y^{m-1} d y \text {. }
$$

Set

$$
\hat{v}=\frac{\sqrt[m-1]{d}}{\sqrt[m-1]{c+\alpha k_{1}}+\sqrt[m-1]{d}}
$$

and we come to

$$
\begin{aligned}
(2) & \geq c(u(x))^{m}+d(x-u(x))^{m}+(m \alpha) \int_{0}^{u(x)}\left\{c \hat{v}^{m}+d(1-\hat{v})^{m}+k_{1} \alpha \hat{v}^{m}\right\} y^{m-1} d y \\
& =c(u(x))^{m}+d(x-u(x))^{m}+\alpha\left\{c \hat{v}^{m}+d(1-\hat{v})^{m}+k_{1} \alpha \hat{v}^{m}\right\} u(x)^{m} d y \\
& =\left\{c u^{m}+d(1-u)^{m}+\alpha\left\{\left(c+k_{1} \alpha\right) \hat{v}^{m}+d(1-\hat{v})^{m}\right\} u^{m}\right\} x^{m} .
\end{aligned}
$$


Set

$$
k_{2}=\left(c+k_{1} \alpha\right) \hat{v}^{m}+d(1-\hat{v})^{m},
$$

and we have finally come to

$$
(3)=\left\{c u^{m}+d(1-u)^{m}+\alpha k_{2} u^{m}\right\} x^{m} .
$$

It is clear that

$$
u=\hat{u}=\frac{\sqrt[m-1]{d}}{\sqrt[m-1]{c+\alpha k_{2}}+\sqrt[m-1]{d}}
$$

minimizes this term. Consequently we can find constants $\hat{w}, \hat{v}$ and $\hat{u}$ using backward induction. This completes the proof of Problem 1.

From now on we only consider the case $m=2$ for the sake of simplicity.

\section{An $n$-stage NDP}

Let us expand the three-stage NDP immediately to an $n$-stage NDP, the formulation of which gives the following problem:

Problem 3-1

$$
\begin{aligned}
& \text { Minimize } \quad c\left(u_{0} x_{0}\right)^{2}+d\left(x_{0}-u_{0} x_{0}\right)^{2} \\
& +\quad 2 \alpha \int_{D_{1}} \frac{c\left(u_{1} x_{1}\right)^{2}+d\left(x_{1}-u_{1} x_{1}\right)^{2}}{x_{1}} d x_{1} \\
& +(2 \alpha)^{2} \iint_{D_{2}} \frac{c\left(u_{2} x_{2}\right)^{2}+d\left(x_{2}-u_{2} x_{2}\right)^{2}}{x_{1} x_{2}} d x_{1} d x_{2} \\
& +\quad \ldots \\
& +\quad(2 \alpha)^{n} \iiint_{-} \frac{c\left(u_{n} x_{n}\right)^{2}+d\left(x_{n}-u_{n} x_{n}\right)^{2}}{x_{1} x_{2} \cdots x_{n}} d x_{1} d x_{2} \cdots d x_{n}
\end{aligned}
$$

subject to $\quad 0<u_{0}, u_{1}, \cdots, u_{n} \leq 1$

where the integral domains are

$$
\begin{aligned}
D_{1} & =\left\{x_{1} \mid 0<x_{1}<u_{0} x_{0}\right\} \subset(0, \infty) \\
D_{2} & =\left\{\left(x_{1}, x_{2}\right) \mid 0<x_{1}<u_{0} x_{0}, 0<x_{1}<u_{1} x_{1}\right\} \subset(0, \infty)^{2} \\
\vdots & \\
D_{n} & =\left\{\left(x_{1}, x_{2}, \cdots, x_{n}\right) \mid 0<x_{1}<u_{0} x_{0}, \cdots, 0<x_{n}<u_{n} x_{n}\right\} \subset(0, \infty)^{n} .
\end{aligned}
$$


This problem is solved as follows. The objective function takes the following form:

$$
\begin{gathered}
c\left(u_{0} x_{0}\right)^{2}+d\left(x_{0}-u_{0} x_{0}\right)^{2}+2 \alpha \int_{0}^{u_{0} x_{0}}\left[\frac{c\left(u_{1} x_{1}\right)^{2}+d\left(x_{1}-u_{1} x_{1}\right)^{2}}{x_{1}}\right. \\
+\frac{2 \alpha}{x_{1}}\left\{\int_{0}^{u_{1} x_{1}} \frac{c\left(u_{2} x_{2}\right)^{2}+d\left(x_{2}-u_{2} x_{2}\right)^{2}}{x_{2}}+\frac{2 \alpha}{x_{2}}\left\{\int_{0}^{u_{2} x_{2}} \frac{c\left(u_{3} x_{3}\right)^{2}+d\left(x_{3}-u_{3} x_{3}\right)^{2}}{x_{3}}\right.\right. \\
+\frac{2 \alpha}{x_{n-2}} \int_{0}^{u_{n-2} x_{n-2}}\left\{\frac{c\left(u_{n-1} x_{n-1}\right)^{2}+d\left(x_{n-1}-u_{n-1} x_{n-1}\right)^{2}}{x_{n-1}}\right. \\
\left.\left.\left.\left.+\frac{2 \alpha}{x_{n-1}} \int_{0}^{u_{n-1} x_{n-1}} \frac{k x_{n}^{2}}{x_{n}} d x_{n}\right\} d x_{n-1} \cdots\right\} d x_{3}\right\} d x_{2}\right] d x_{1} .
\end{gathered}
$$

Calculating the integrals backward from

$$
\int_{0}^{u_{n-1} x_{n-1}} \frac{k x_{n}^{2}}{x_{n}} d x_{n}
$$

we have

$$
\begin{aligned}
x_{0}^{2}\left[c\left(u_{0}\right)^{2}+d(1-\right. & \left.u_{0}\right)^{2}+\alpha u_{0}^{2}\left\{c\left(u_{1}\right)^{2}+d\left(1-u_{1}\right)^{2}\right. \\
+\alpha u_{1}^{2}\left\{c\left(u_{2}\right)^{2}+d\right. & \left(1-u_{2}\right)^{2}+\alpha u_{2}^{2}\left\{c\left(u_{3}\right)^{2}+\cdots\right. \\
& \left.+\alpha u_{n-2}^{2}\left\{c u_{n-1}^{2}+d\left(1-u_{n-1}\right)^{2}+k \alpha u_{n-1}^{2}\right\}\right] .
\end{aligned}
$$

Consequently, calculating $k_{1}, k_{2}, \cdots, k_{n-1}, k_{n}$ in the similar manner as 3 -stage NDP, we set the minimum point to $k_{n}$ and the minima to

$$
\hat{u}_{i}=\frac{d}{c+d+\alpha k_{n-1}} \quad(1 \leq i \leq n)
$$

where $k_{0}=k$. Thus we have solved the problem of minimization of $n+1$ functions.

\section{Infinite-stage NDP}

In this section, we take up a problem of minimization $P\left(x_{0}\right)$ of countably infinite functions

$$
u_{0}=u_{0}\left(x_{0}\right), u_{1}=u_{1}\left(x_{1}\right), \cdots, u_{n}=u_{n}\left(x_{n}\right), \cdots
$$


Problem 4-1

$$
\begin{aligned}
\text { Minimize } & c\left(u_{0} x_{0}\right)^{2}+d\left(x_{0}-u_{0} x_{0}\right)^{2} \\
& +2 \alpha \int_{D_{1}} \frac{c\left(u_{1} x_{1}\right)^{2}+d\left(x_{1}-u_{1} x_{1}\right)^{2}}{x_{1}} d x_{1} \\
+ & (2 \alpha)^{2} \iint_{D_{2}} \frac{c\left(u_{2} x_{2}\right)^{2}+d\left(x_{2}-u_{2} x_{2}\right)^{2}}{x_{1} x_{2}} d x_{1} d x_{2} \\
+ & \cdots \\
+ & (2 \alpha)^{n} \iiint_{D_{n}} \frac{c\left(u_{n} x_{n}\right)^{2}+d\left(x_{n}-u_{n} x_{n}\right)^{2}}{x_{1} x_{2} \cdots x_{n}} d x_{1} d x_{2} \cdots d x_{n} \\
+ &
\end{aligned}
$$

subject to $0<u_{0}, u_{1}, \cdots, u_{n}, \cdots \leq 1$

where the integral domains are

$$
\begin{aligned}
& D_{1}=\left\{x_{1} \mid 0<x_{1}<u_{0} x_{0}\right\} \subset(0, \infty) \\
& D_{2}=\left\{\left(x_{1}, x_{2}\right) \mid 0<x_{1}<u_{0} x_{0}, 0<x_{1}<u_{1} x_{1}\right\} \subset(0, \infty)^{2} \\
& D_{n}=\left\{\left(x_{1}, x_{2}, \cdots, x_{n}\right) \mid 0<x_{1}<u_{0} x_{0}, 0<x_{1}<u_{1} x_{1},\right. \\
& \left.\cdots, 0<x_{n}<u_{n} x_{n-1}\right\} \subset(0, \infty)^{n}
\end{aligned}
$$

In this situation we call this series of minimal functions optimal policy and $x_{0}$ the initial state. When we consider the minima

$$
J\left(x_{0}\right)=J\left(x_{0} ; c, d, \alpha\right)
$$

to be a function of the initial state $x_{0}$, we call $J$ a minimum value function, an optimal function or a value function. The dynamics of this infinite stage decision-making is non-deterministic in the following sense.

When we choose the decision $u_{n}\left(\in\left(0, x_{n}\right]\right)$ in a state $x_{n}(\in(0, \infty))$ at time $n$, it does not necessarily transit to a state $x_{n+1}$ determined uniquely by the state $x_{n}$ and the decision $u_{n}$ at time $n+1$, but does to any state $x_{n+1}$ in the half open interval $\left(0, u_{n}\right]$ with the weight

$$
\beta\left(x_{n}, u_{n}, x_{n+1}\right)=\frac{2 \alpha}{x_{n+1}}(>0) .
$$

Then, the total of the possible transition weights is indicated by

$$
\int_{0}^{u_{n}} \beta\left(x_{n}, u_{n}, x_{n+1}\right) d x_{n+1}=2 \alpha \int_{0}^{u_{n}} \frac{d x_{n+1}}{x_{n+1}} .
$$

This value is never finite and divergent to $\infty$. Therefore, this transit rule is not stochastic. Under the stochastic transition rule, the summation of the probability of transiting to 
possible next states is finite and becomes one with appropriate normalization. In three transition rulees the following inclusive relation holds:

deterministic $\subset$ stochastic $\subset$ non deterministic

In this paper, non-deterministic transition means a transit to one state or more with appropriate nonnegative weight(s). A transition law where the total of weights is 1 is called stochastic. And the transition weight is called a transition probability. A transition law where the total of weights is not finite is called non-deterministic.

An $n$-th decision function prescribes the way to select a decision at time $n$. This function is indicated as

$$
f_{n}:(0, \infty) \rightarrow(0, \infty)
$$

which satisfies

$$
0<f_{n}(x) \leq x \quad \text { for any } x \in(0, \infty)
$$

We call a series of decision functions

$$
\pi=\left\{f_{0}, f_{1}, \cdots, f_{n}, \cdots\right\}
$$

a policy or Markov policy. We indicate the whole policies as $\Pi$ and call Markov policy class or policy class in short.

Suppose now that a decision maker adopts a policy

$$
\pi=\left\{f_{0}, f_{1}, \cdots, f_{n}, \cdots\right\}(\in \Pi) .
$$

Then, on state of $x_{n} \in(0, \infty)$ at time $n$ the decision

$$
u_{n}=f\left(x_{n}\right)\left(\in\left(0, x_{n}\right]\right)
$$

is selected by $n$-th decision function $f_{n}$. This decision incurs a quadratic cost

$$
r_{n}=r_{n}\left(x_{n}, u_{n}\right)=c u_{n}^{2}+d\left(x_{n}-u_{n}\right)^{2} .
$$

However, this cost is evaluated by the product

$$
\beta_{0} \beta_{1} \cdots \beta_{n-1} r_{n}=\frac{(2 \alpha)^{n}\left[c u_{n}^{2}+d\left(x_{n}-u_{n}\right)^{2}\right]}{x_{1} x_{2} \cdots x_{n}}
$$

of the weight related to the path $\left(x_{0}, u_{0}, x_{1}, u_{1}, x_{n-1}, u_{n-1}, x_{n}\right)$ from the initial state $x_{0}$ to $x_{n}$

$$
\beta_{0} \beta_{1} \cdots \beta_{n-1}=\frac{2 \alpha}{x_{1}} \frac{2 \alpha}{x_{2}} \cdots \frac{2 \alpha}{x_{n}}=\frac{(2 \alpha)^{n}}{x_{1} x_{2} \cdots x_{n}}\left(\beta_{m}=\beta\left(x_{m}, u_{m}, x_{m+1}\right)\right) .
$$

The summation of the value over the whole possible paths is indicated as $n$-th iterated integral:

$$
\begin{aligned}
W_{x_{0}}\left[r_{n}\right] & =\iint \cdots \int_{R} \beta_{0} \beta_{1} \cdots \beta_{n-1} r_{n} d x_{1} d x_{2} \cdots d x_{n} \\
& =\iint \cdots \int_{R} \frac{(2 \alpha)^{n}\left[c u_{n}^{2}+d\left(x_{n}-u_{n}\right)^{2}\right]}{x_{1} x_{2} \cdots x_{n}} d x_{1} d x_{2} \cdots d x_{n}
\end{aligned}
$$


where the integral domain is determined by a series of decision functions $f_{0}, f_{1}, \cdots, f_{n-1}$ as follows :

$$
R=\left\{\left(x_{1}, x_{2}, \cdots, x_{n}\right) \mid 0<x_{1}<u_{0}, 0<x_{2}<u_{1}, \cdots, 0<x_{n}<u_{n-1}\right\} \subset(0, \infty)^{n} .
$$

Here $u_{m}=f_{m}\left(x_{m}\right) m=0,1, \cdots, n-1$. Especially when $n=0$,

$$
W_{x_{0}}\left[r_{0}\right]=c u_{0}^{2}+d\left(x_{0}-u_{0}\right)^{2} .
$$

The total summation of $m$-th iterated integral for all $m=0,1, \cdots, n, \cdots$

$$
J\left(x_{0} ; \pi\right)=W_{x_{0}}\left[r_{0}\right]+W_{x_{0}}\left[r_{1}\right]+\cdots+W_{x_{0}}\left[r_{n}\right]+\cdots
$$

is a function of the initial state $x_{0}$ and policy $\pi$. This is called the total weighted cost from the initial state $x_{0}$ with the policy $\pi$. An optimization problem is to select a policy which minimizes the total weighted cost for given initial state. The problem is represented as the following minimization problem:

$$
P\left(x_{0}\right) \quad \text { minimize } J\left(x_{0} ; \pi\right) \text { subject to } \pi \in \Pi
$$

As is well-known in DP theory, the minimun value function

$$
v(x)=J(x ; \hat{\pi}):(0, \infty) \rightarrow R^{1}
$$

satisfies the following Bellman equation (4), where $\hat{\pi}$ is an optimal policy. In stead of solving $P\left(x_{0}\right)$, we solve the following equivalent problem.

Problem 4-2 [Controlled integral equation (Iwamoto)]

Let $c>0, d>0$ and $0<\alpha<1$ be constant. Find a pair of a function $v(x)$ and the minimal function $u(x)$, which satisfy the controlled integral equation

$$
v(x)=\min _{0<u<x}\left[c u^{2}+d(x-u)^{2}+\int_{0}^{u} \frac{2 \alpha v(y)}{y} d y\right] \quad x>0 .
$$

We solve this problem for case $c=d=\alpha=1$ using the method of successive approximation.

Let us solve the iterated equation

$$
v_{n+1}(x)=\min _{0<u<x}\left[c u^{2}+d(x-u)^{2}+\int_{0}^{u} \frac{2 \alpha v_{n}(y)}{y} d y\right] \quad v_{0}(x)=0 .
$$

First we have

$$
\begin{aligned}
& v_{1}(x)=\min _{0 \leq u \leq x}\left[u^{2}+(x-u)^{2}\right]=2\left(\frac{x}{2}\right)^{2}=\frac{x^{2}}{2} \\
& v_{2}(x)=\min _{0 \leq u \leq x}\left[u^{2}+(x-u)^{2}+2 \int_{0}^{u} \frac{y}{2} d y\right]=\min _{0 \leq u \leq x}\left[\frac{3 u^{2}}{2}+(x-u)^{2}\right]=\frac{3}{5} x^{2} \\
& v_{3}(x)=\min _{0 \leq u \leq x}\left[u^{2}+(x-u)^{2}+2 \int_{0}^{u} \frac{3 y}{5} d y\right]=\min _{0 \leq u \leq x}\left[\frac{8 u^{2}}{5}+(x-u)^{2}\right]=\frac{8}{13} x^{2} .
\end{aligned}
$$


Second

$$
v_{n+1}(x)=v_{n+1} x^{2}=\min _{0 \leq u \leq x}\left[u^{2}+(x-u)^{2}+2 \int_{0}^{u} v_{n} y d y\right]=\frac{v_{n}+1}{v_{n}+2} x^{2}
$$

yields

$$
v_{n+1}=\frac{v_{n}+1}{v_{n}+2}, \quad u_{n+1}=\frac{1}{v_{n}+2} .
$$

Finally setting

$$
\lim _{n \rightarrow \infty} v_{n}=\alpha
$$

we have

$$
\alpha=\frac{\alpha+1}{\alpha+2} .
$$

This implies

$$
\alpha=\frac{-1+\sqrt{5}}{2}=v(\alpha>0)
$$

Moreover we have

$$
\lim _{n \rightarrow \infty} u_{n}=\frac{1}{\lim _{n \rightarrow \infty} v_{n}+2}=\frac{3-\sqrt{5}}{2}=u \fallingdotseq 0.38196
$$

In this manner we can solve the original control integral equation by the method of successive approximation. As $v(x)$ is a solution of the original control integral equation, we come to the the following theorem:

THEOREM 4.1. The quadratic function $v(x)=v x^{2}\left(v=\frac{-1+\sqrt{5}}{2}\right)$ is a solution of the equation:

$$
(\star) \quad v(x)=\min _{0 \leq u \leq x}\left[u^{2}+(x-u)^{2}+2 \int_{0}^{u} \frac{v(y)}{y} d y\right] \quad x \geq 0 .
$$

Proof

When $v(x)=v x^{2}$, we get

$$
2 \int_{0}^{u} \frac{v(y)}{y} d y=\frac{1+\sqrt{5}}{2} u^{2}+(x-u)^{2}
$$

Differentiating this equation in regard to $u$ and setting the derivative to zero, we have

$$
u=\frac{3-\sqrt{5}}{2} x
$$

Therefore,

$$
\min _{0 \leq u \leq x}\left[u^{2}+(x-u)^{2}+2 \int_{0}^{u} \frac{v(y)}{y} d y\right]=\frac{-1+\sqrt{5}}{2} x^{2}
$$


The last term is equal to $v(x)$, and the minimal function is

$$
\begin{aligned}
\hat{u}(x) & =\frac{3-\sqrt{5}}{2} x . \\
v & \fallingdotseq 0.61803 .
\end{aligned}
$$

The following theorem is also valid:

TheOREM 4.2. The control integral equation in Problem 4-1 is equivalent to the following problem:

$$
\min _{0 \leq u<1}\left[\frac{u^{2}+(1-u)^{2}}{1-u^{2}}\right] .
$$

Proof. From Problem 4.1,

$$
\begin{aligned}
& \left(u x_{0}\right)^{2}+\left(x_{0}-u x_{0}\right)^{2}+2 \int_{D_{1}} \frac{\left(u x_{1}\right)^{2}+\left(x_{1}-u x_{1}\right)^{2}}{x_{1}} d x_{1} \\
+ & 2^{2} \iint_{D_{2}} \frac{\left(u x_{2}\right)^{2}+\left(x_{2}-u x_{2}\right)^{2}}{x_{1} x_{2}} d x_{1} d x_{2}+2^{3} \iiint_{D_{3}} \frac{\left(u x_{3}\right)^{2}+\left(x_{3}-u x_{3}\right)^{2}}{x_{1} x_{2} x_{3}} d x_{1} d x_{2} d x_{3} \\
& +\cdots+2^{n} \iiint \cdots \int_{D_{n}} \frac{\left(u x_{n}\right)^{2}+\left(x_{n}-u x_{n}\right)^{2}}{x_{1} x_{2} x_{3} \cdots x_{n}} d x_{1} d x_{2} d x_{3} \cdots d x_{n}+\cdots \\
= & \left\{u^{2}+(1-u)^{2}\right\} x_{0}^{2}+\left\{u^{2}+(1-u)^{2}\right\}\left(u x_{0}\right)^{2}+\left\{u^{2}+(1-u)^{2}\right\}\left(u^{2} x_{0}\right)^{2}+\cdots \\
= & \left\{u^{2}+(1-u)^{2}\right\} x_{0}^{2} \sum_{i=0}^{\infty} u^{2 i}=\frac{u^{2}+(1-u)^{2}}{1-u^{2}} x_{0}^{2} .
\end{aligned}
$$

Solution

$$
\frac{d}{d u}\left[\frac{u^{2}+(1-u)^{2}}{1-u^{2}}\right]=\frac{-2 u^{2}+6 u-2}{\left(1-u^{2}\right)^{2}} .
$$

Setting the numerator to zero, we have

$$
u=\frac{3-\sqrt{5}}{2}(0<u<1) \text {. }
$$

\section{NDP with discount factor}

We can extend the problem considered in Section 4 further with introduction of discount factor $\beta$.

Problem 5-1

$$
v(x)=\max _{0 \leq u \leq x}\left[u^{s}+(x-u)^{s}+s \beta \int_{0}^{u} \frac{v(y)}{y} d y\right] \quad x \geq 0,0<s<1 .
$$


To solve this, we use the method of successive approximation.

Define the function $v_{n+1}(x)$ to be

$$
v_{n+1}(x)=\max _{0 \leq u \leq x}\left[u^{s}+(x-u)^{s}+s \beta \int_{0}^{u} \frac{v_{n}(y)}{y} d y\right] \quad v_{0}(x)=0 .
$$

First

$$
v_{1}(x)=\max _{0 \leq u \leq x}\left[u^{s}+(x-u)^{s}\right]=\left(\frac{x}{2}\right)^{s}+\left(\frac{x}{2}\right)^{s}=2^{1-s} x^{s}
$$

Then setting $v_{n}(x)=v_{n} x^{s}$, we have

$$
\begin{aligned}
v_{n+1} x^{s} & =\max _{0 \leq u \leq x}\left[u^{s}+(x-u)^{s}+s \beta \int_{0}^{u} \frac{v_{n} y^{s}}{y} d y\right] \\
& =\max _{0 \leq u \leq x}\left[\left(\beta v_{n}+1\right) u^{s}+(x-u)^{s}\right] .
\end{aligned}
$$

Differentiating $f(u) \equiv\left(\beta v_{n}+1\right) u^{s}+(x-u)^{s}$ in regard to $u$ and setting the derivative to zero, we get

$$
u=\frac{1}{\left(\beta v_{n}+1\right)^{\frac{1}{s-1}}+1} x .
$$

When we set $\lim _{n \rightarrow \infty} v_{n}=v, \lim _{n \rightarrow \infty} u_{n}=u$, we have

$$
v=\frac{(\beta v+1)}{\left((\beta v+1)^{\frac{1}{s-1}}+1\right)^{s-1}}, \quad\left((\beta v+1)^{\frac{1}{s-1}}+1\right)^{s-1}=\frac{\beta v+1}{v} .
$$

Now we set $f(v)=\left((\beta v+1)^{\frac{1}{s-1}}+1\right)^{s-1}-\frac{\beta v+1}{v}$ and $t=(\beta v+1)^{\frac{1}{s-1}}$.

$$
g(t)=(t+1)^{s-1}-\frac{t^{s-1}}{\frac{\left(t^{s-1}-1\right)}{\beta}}=(t+1)^{s-1}-\frac{\beta t^{s-1}}{\left(t^{s-1}-1\right)} .
$$

Then

$$
g^{\prime}(t)=(s-1)\left\{(t+1)^{s-2}+\frac{\beta t^{s-2}}{\left(t^{s-1}-1\right)^{2}}\right\}<0
$$

Since $g(t)$ is a decreasing function and

$$
g(0)=1, \lim _{t \rightarrow 1-0} g(t)=-\infty
$$

there exists only one $t$ which satisfies $g(t)=0$ in $0<t<1$. As $t=(\beta v+1)^{\frac{1}{s-1}}$, we have

$$
v=\frac{t^{s-1}-1}{\beta} .
$$




\section{Consideration on weight function}

So far we have only considered

$$
\frac{1}{z}
$$

as a weight function. We wonder if there are other weight functions that are suitable for recursive equations. We should consider the necessary and sufficient conditions for

$$
\int_{0}^{u} \frac{f(y)}{g(y)} d y
$$

to be represented in the form of $f(u)$. Since it is sufficient that

$$
\int_{0}^{x} \frac{f(y)}{g(y)} d y=k f(x)
$$

we differentiate the both sides of the equation to get

$$
\frac{f(x)}{g(x)}=k f^{\prime}(x)
$$

When

$$
f(x) \neq 0,
$$

we have

$$
\frac{f^{\prime}(x)}{f(x)}=\frac{1}{k g(x)} .
$$

Now let's consider separately the cases depending on the type of $g(x)$. In this section $C_{1}, C_{2}, C_{3}$ and $C_{4}$ are constants.

(1) $g(x)=c($ constant $)$

$$
\{\log f(x)\}^{\prime}=C_{1} \quad \log f(x)=C_{1} x+C_{2} \quad f(x)=C_{3} e^{C_{1} x} .
$$

(2) $g(x)=x$

$$
\{\log f(x)\}^{\prime}=\frac{C_{1}}{x} \quad \log f(x)=C_{1} \log x+C_{2} \quad f(x)=C_{3} x^{C_{1}} .
$$

(3) $g(x)=x^{\alpha}$

$$
\{\log f(x)\}^{\prime}=\frac{C_{1}}{x^{\alpha}} \quad \alpha \neq 1 \rightarrow \log f(x)=C_{1} x^{-\alpha+1}+C_{2} \quad f(x)=C_{3} e^{C_{1} x^{-\alpha+1}} .
$$

(4) $g(x)=e^{x}$

$$
\{\log f(x)\}^{\prime}=C_{1} e^{-x} \quad \log f(x)=-C_{1} e^{-x}+C_{2} \quad f(x)=C_{3} e^{C_{4} e^{-x}} .
$$


(5) $g(x)=x \log x$

$$
\{\log f(x)\}^{\prime}=\frac{C_{1}}{x \log x} \quad \log f(x)=C_{1} \log (\log x)+C_{2} \quad f(x)=C_{3}(\log x)^{C_{4}} .
$$

(6) $g(x)=\tan x$

$$
\{\log f(x)\}^{\prime}=C_{1} \cot x \quad \log f(x)=C_{1} \log (\sin x)+C_{2} \quad f(x)=C_{3}(\sin x)^{C_{4}} .
$$

(7) $g(x)=\cot x$

$$
\{\log f(x)\}^{\prime}=C_{1} \tan x \quad \log f(x)=-C_{1} \log (\cos x)+C_{2} \quad f(x)=C_{3}(\cos x)^{C_{4}} .
$$

Among these cases, the weight becomes $\infty$ at $x=0$ only in cases (2), (3) and (6). We examine the case (6).

When $g(x)=\tan x, f(x)=\sin x$.

$$
v(x)=\min _{0 \leq u \leq x}\left[\sin u+\sin (x-u)+\int_{0}^{u} \frac{\sin y}{\tan y} d y\right] \quad x \geq 0 .
$$

We apply the method of successive approximation to solve this. Define the function $v_{n+1}(x)$ to be

$$
\begin{aligned}
& v_{n+1}(x)=\min _{0 \leq u \leq x}\left[\sin u+\sin (x-u)+\int_{0}^{u} \frac{v_{n}(y)}{\tan y} d y\right] \quad x \geq 0 . \\
& v_{1}(x)=\min _{0 \leq u \leq x}[\sin u+\sin (x-u)]=\sin \frac{x}{2}+\sin \frac{x}{2}=2 \sin \frac{x}{2} .
\end{aligned}
$$

As above trigonometric functions are not suitable because the form of the functions changes after calculation. We examine the case(3).

$$
\text { When } g(x)=x^{\alpha}(\alpha \neq 1), \quad f(x)=C_{3} e^{C_{1} x^{-\alpha+1}} .
$$

If we set $f(x)=e^{-x^{-\alpha+1}}$ then

$$
v(x)=\max _{0 \leq u \leq x}\left[e^{-u^{-\alpha+1}}+e^{-(x-u)^{-\alpha+1}}+\int_{0}^{u} \frac{v(y)}{y^{\alpha}} d y\right] \quad x \geq 0 .
$$

It is clear that exponential functions are not suitable. From the above examination, we should conclude that there is no weight function other than

$$
g(x)=\frac{1}{x} .
$$




\section{References}

Bellman, R.E. (1957). Dynamic Programming, Princeton Univ. Press, New Jersey.

Hisano, H. (2002). Optimal stopping problem on finite Markov chain, Bull. Inform. Cybernet. 34 , no. $2,97-104$.

Hisano, H. (2007). An Optimal stopping problem on tree, Bull. Inform. Cybernet. 39, 45-57.

Fujita, T., Ueno, T. and Iwamoto, S. (2004). A nondeterministic dynamic programming model, Lecture Notes in Artificial Intelligence 3214, 1208-1214.

Iwamoto, S. (2005). Richard E. Bellman: Dynamic Programming; Prominent Books and Articles in the 20th Century, (in Japanese), Information Processing 46, no.7, pp.842.

Received October 10, 2007

Revised May 3, 2008 